\title{
On Existence of Local Martingale Measures for Insiders who Can Stop at Honest Times
} by

\author{
Jakub ZWIERZ
}

Presented by Jerzy $Z A B C Z Y K$

\begin{abstract}
Summary. We consider a market with two types of agents with different levels of information. In addition to a regular agent, there is an insider whose additional knowledge consists of being able to stop at an honest time $\Lambda$. We show, using the multiplicative decomposition of the Azéma supermartingale, that if the martingale part of the price process has the predictable representation property and $\Lambda$ satisfies some mild assumptions, then there is no equivalent local martingale measure for the insider. This extends the results obtained by Imkeller to the continuous semimartingale setting and general honest times.
\end{abstract}

1. Introduction. We shall consider the model of financial market with two types of participants: a regular trader, and a second investor (insider) who is in possession of more knowledge than the regular trader. The flow of information for both types of market participants is modeled by the underlying filtrations. While the regular trader's filtration $\mathbb{F}=\left(\mathcal{F}_{t}\right)_{t \geq 0}$ is assumed to coincide with the filtration generated by the stock price process, the insider's filtration $\mathbb{G}=\left(\mathcal{G}_{t}\right)_{t \geq 0}$ is strictly larger, and may be written in the form $\mathcal{G}_{t}=\bigcap_{\varepsilon>0}\left(\mathcal{F}_{t+\varepsilon} \vee \mathcal{H}_{t+\varepsilon}\right)$, where $\mathbb{H}=\left(\mathcal{H}_{t}\right)_{t \geq 0}$ is a filtration representing the insider's additional information. The main technique used in this context is the grossissement de filtration, developed in a series of works by Jeulin (see [6]), Jacod and Yor (see [7]) and others. We will focus on the case where $\mathcal{H}_{t}=\sigma(\Lambda \wedge t)$ for some r.v. $\Lambda$, i.e. $\mathbb{G}$ is the smallest filtration containing $\mathbb{F}$ and making $\Lambda$ a $\mathbb{G}$-stopping time. In other words, we assume that the insider is able to stop at some random time $\Lambda$, which is not available to the regular trader. Within this framework we shall study the problem of

2000 Mathematics Subject Classification: Primary 60H30; Secondary 60G44.

Key words and phrases: insider trading, progressive enlargement of filtrations, equivalent martingale measure, NFLVR, honest time. 
existence of equivalent local martingale measures for the insider, which is also closely related to the existence of arbitrage opportunities. We show that under the assumption that all martingales with respect to $\mathbb{F}$ are continuous and $\Lambda$ is an honest time (that is, the "end of a predictable set" in an appropriate sense) avoiding $\mathbb{F}$-stopping times, if the price process enjoys the predictable representation property, then there is no measure $\mathbb{Q}$ equivalent to $\mathbb{P}$ and making the discounted price process a $(\mathbb{G}, \mathbb{Q})$-local martingale. The main tool in the proof is the multiplicative representation of the Azéma supermartingale given in [9]. Thus, we extend the results obtained in [5] to the semimartingale framework and general honest times. We shall also give an explicit example of an arbitrage opportunity arising for a particular $\Lambda$.

2. Market model and preliminaries. Throughout this paper we will work with the probability space $(\Omega, \mathcal{F}, \mathbb{F}, \mathbb{P})$, where $\mathbb{F}=\left(\mathcal{F}_{t}\right)_{t \in[0, T]}$ is a filtration satisfying the usual conditions, and $T$ is some finite time horizon (we may assume that $\mathcal{F}_{s}=\mathcal{F}_{T}$ for $s>T$ ). Further, let $S=\left(S_{t}\right)_{t \in[0, T]}$ be a 1-dimensional continuous semimartingale representing the discounted asset price. We shall assume that the filtration $\mathbb{F}$ is generated by $S$. Moreover, we assume that the market defined by $S$ satisfies the (NFLVR) (no free lunch with vanishing risk) condition. Let us briefly recall its definition (see e.g. [3] for details). We say that the strategy $\theta$ is a-admissible for $a \in \mathbb{R}_{+}$if $\theta_{0}=0$, and for all $t \in[0, T], \int_{0}^{t} \theta_{u} d S_{u} \geq-a \mathbb{P}$-a.s. If there exists some $a \in \mathbb{R}_{+}$for which $\theta$ is $a$-admissible, we will call it simply admissible.

Definition 2.1. The process $\left(S_{t}\right)_{t \in[0, T]}$ satisfies the (NFLVR) condition if for every sequence $\left\{\phi_{n}\right\}$ of simple $\left(^{1}\right)$ predictable processes which are $\delta_{n^{-}}$ admissible with $\delta_{n} \rightarrow 0$, we have $V_{T}\left(\phi_{n}\right) \rightarrow 0$ in $\mathbb{P}$, where $V_{T}(\phi)$ denotes the value process for the strategy $\phi$ at time $T$.

In the paper of Delbaen and Schachermayer [4] it was shown that if the market satisfies the (NFLVR) condition, then the Doob-Meyer decomposition of $S$ is given by the formula

$$
S_{t}=M_{t}+\int_{0}^{t} \alpha_{s} d\langle M, M\rangle_{s},
$$

where $M=\left(M_{t}\right)_{t \in[0, T]}$ is the martingale part of $S$, and $\alpha=\left(\alpha_{t}\right)_{t \in[0, T]}$ is an $\mathbb{F}$-predictable process. Another well known fact, proved in the fundamental paper [3], is that the (NFLVR) property of $S$ is a necessary and sufficient condition for the existence of an equivalent local martingale measure $\mathbb{Q}$.

$\left({ }^{1}\right)$ An $\mathbb{F}$-predictable process $\phi$ is called simple if it is a linear combination of elements of the form $D 1_{\left[T_{1}, T_{2}\right]}$, where $T_{1} \leq T_{2}$ are $\mathbb{F}$-stopping times, $D$ is $\mathcal{F}_{T_{1}}$-measurable, and ]$\left.T_{1}, T_{2}\right]=\left\{(t, \omega): t<\infty, T_{1}(\omega)<t \leq T_{2}(\omega)\right\}$. 
Thus, we assume that the discounted price process is of the form (2.1), and that there exists a local martingale measure $\mathbb{Q} \sim \mathbb{P}$ or equivalently that $S$ satisfies the (NFLVR).

On the other hand, as a consequence of Lemma 3.8 in [4] (see also Corollary 2.4 in [2]) we have the following theorem:

Theorem 2.2. If $\mathbb{P}\left(\int_{0}^{T} \alpha_{t}^{2} d\langle M, M\rangle_{t}=\infty\right)>0$, then $S$ does not satisfy the (NFLVR) condition.

The above condition will be crucial for our investigation of nonexistence of equivalent local martingale measures.

Now, we turn to the description of the insider's view of the market. As mentioned in the introduction, we assume that the insider is able to stop at a random time $\Lambda$, that is, his filtration $\mathbb{G}=\left(\mathcal{G}_{t}\right)_{t \in[0, T]}$ is given by $\mathcal{G}_{t}=\bigcap_{\varepsilon>0}\left(\mathcal{F}_{t+\varepsilon} \vee \sigma(\Lambda \wedge(t+\varepsilon))\right)$. Of course, a natural question arises whether $S$ is still a $(\mathbb{G}, \mathbb{P})$-semimartingale. It turns out that the answer is positive if we restrict the admissible $\Lambda$ 's to so called honest times. More precisely, we have the following definition:

DEFINITION 2.3. $\Lambda$ is called an honest time if there exists a predictable set $\Sigma \subset[0, T] \times \Omega$ such that $\Lambda(\omega)=\sup \{t \geq 0:(t, \omega) \in \Sigma\}$.

A crucial object needed to deal with the properties of $S$ in the enlarged filtration is the Azéma supermartingale $Z=\left(Z_{t}\right)_{t \geq 0}$ defined as $\left({ }^{2}\right)$ $Z_{t}=\mathbb{P}\left(\Lambda>t \mid \mathcal{F}_{t}\right)$. Now, the following theorem provides the Doob-Meyer decomposition of $S$ in the filtration $\mathbb{G}$ (see [6]).

Theorem 2.4. Suppose that $M=\left(M_{t}\right)_{t \geq 0}$ is an $(\mathbb{F}, \mathbb{P})$-local martingale, and $\Lambda$ is an honest time. Then there exists a $(\mathbb{G}, \mathbb{P})$-local martingale $\widehat{M}=$ $\left(\widehat{M}_{t}\right)_{t \geq 0}$ such that $\left(^{3}\right)$

$$
M_{t}=\widehat{M}_{t}+\int_{0}^{t \wedge \Lambda} \frac{d\langle M, Z\rangle_{s}}{Z_{s-}}+\int_{\Lambda}^{t} \frac{d\langle M, 1-Z\rangle_{s}}{1-Z_{s-}} .
$$

In particular, $M$ is a $(\mathbb{G}, \mathbb{P})$-semimartingale.

The question we shall deal with in this paper is the existence of local martingale measures for the insider, i.e. measures $\mathbb{Q} \sim \mathbb{P}$ such that $S$ is the $(\mathbb{G}, \mathbb{Q})$-local martingale. However, the setting provided by Theorem 2.4 is not particularly well suited to address this problem, as it is generally hard to compute the Azéma supermartingale directly from the definition (see [5] for several examples). We solve this difficulty by using the multiplicative representation of $Z$ given by Nikeghbali and Yor in [9]. We will recall here briefly

$\left(^{2}\right)$ We will consider a càdlàg version of this supermartingale.

$\left(^{3}\right)$ We adopt the convention that $\int_{s}^{t} \nu_{u} d H_{u}=0$ for $s \geq t$. 
the most important result of that paper. The following two assumptions will be needed:

(C) All $\mathbb{F}$-martingales are continuous.

(A) $\Lambda$ avoids $\mathbb{F}$-stopping times, that is, for every $\mathbb{F}$-stopping time $V$ we have $\mathbb{P}(\Lambda=V)=0$.

In the literature, conditions (CA) are usually assumed to hold for progressive enlargements. Now, we have the following theorem:

Theorem 2.5 ([9, Theorem 4.1]). Let $\Lambda$ be an $\mathbb{F}$-honest time. Then, under conditions $(\mathbf{C A})$, there exists a continuous and nonnegative $(\mathbb{F}, \mathbb{P})$ local martingale $N=\left(N_{t}\right)_{t \geq 0}$, with $N_{0}=1$ and $\lim _{t \rightarrow \infty} N_{t}=0$, such that

$$
Z_{t}=\frac{N_{t}}{\bar{N}_{t}}
$$

where $\bar{N}_{t}=\sup _{s \leq t} N_{s}$.

We can also give a description of $\Lambda$ in terms of $N$. It follows easily from considerations in [9], but since it is not explicitly stated there, we will give the proof for the sake of completeness.

Theorem 2.6. $\Lambda=\sup \left\{t \geq 0: N_{t}=\bar{N}_{t}\right\}$.

Proof. From the preceding theorem we get

$$
\mathbb{P}\left(\Lambda>t \mid \mathcal{F}_{t}\right)=N_{t} / \bar{N}_{t}
$$

On the other hand, $\mathbb{P}\left(\sup _{s>t} N_{s}>\bar{N}_{t} \mid \mathcal{F}_{t}\right)=N_{t} / \bar{N}_{t}$ (see [8, Lemma 0.1]) and thus $\{\Lambda>t\}=\left\{\sup _{s>t} N_{s}>\bar{N}_{t}\right\}=\left\{\exists_{u>t} N_{u}=\bar{N}_{\infty}\right\} \mathbb{P}$-a.s., which yields the result.

3. Martingale measures for the insider. We are now ready to state the main result. The additional assumption we will need is that the martingale part of $S$, i.e. $M$, has the predictable representation property, that is, for every $(\mathbb{F}, \mathbb{P})$-local martingale $N$, there exists $c \in \mathbb{R}$ and an $\mathbb{F}$-predictable process $n=\left(n_{t}\right)_{t \geq 0}$ such that for all $t \in[0, T], \int_{0}^{t} n_{s}^{2} d\langle M, M\rangle_{s}<\infty$ and

$$
N_{t}=c+\int_{0}^{t} n_{s} d M_{s} .
$$

Then we have the following theorem.

THEOREM 3.1. Let $S=\left(S_{t}\right)_{t \in[0, T]}$ be a continuous $(\mathbb{F}, \mathbb{P})$-semimartingale satisfying the $(N F L V R)$ condition, and such that $M$, the martingale part of $S$, has the predictable representation property. Suppose that $\Lambda$ is an $\mathbb{F}$ honest time such that $\mathbb{P}(\Lambda<T)=1$, and conditions (CA) are satisfied. Then there is no equivalent local martingale measure for $S$ in the filtration $\mathbb{G}=\left(\mathcal{G}_{t}\right)_{t \in[0, T]}$, where $\mathcal{G}_{t}=\bigcap_{\varepsilon>0}\left(\mathcal{F}_{t+\varepsilon} \vee \sigma(\Lambda \wedge(t+\varepsilon))\right)$. 
Proof. Let $Z=\left(Z_{t}\right)_{t \geq 0}$ be the Azéma supermartingale associated with $\Lambda$, and $N=\left(N_{t}\right)_{t \geq 0}$ be the $(\mathbb{F}, \mathbb{P})$-local martingale from its multiplicative decomposition, i.e. $Z_{t}=N_{t} / \bar{N}_{t}, \bar{N}_{t}=\sup _{s<t} N_{s}$. From Itô's formula we get

$$
d Z_{t}=\frac{d N_{t}}{\bar{N}_{t}}-\frac{N_{t}}{\bar{N}_{t}^{2}} d \bar{N}_{t}
$$

Therefore

$$
\langle M, Z\rangle_{t}=\int_{0}^{t} \frac{d\langle N, M\rangle_{u}}{\bar{N}_{u}} .
$$

Thus, the Doob-Meyer decomposition of $M$ with respect to the enlarged filtration takes the form

$$
\begin{aligned}
M_{t} & =\widehat{M}_{t}+\int_{0}^{t \wedge \Lambda} \frac{d\langle N, M\rangle_{u}}{\bar{N}_{u} \frac{N_{u}}{\bar{N}_{u}}}-\int_{\Lambda}^{t} \frac{d\langle N, M\rangle_{u}}{\bar{N}_{u}\left(1-\frac{N_{u}}{\bar{N}_{u}}\right)} \\
& =\widehat{M}_{t}+\int_{0}^{t \wedge \Lambda} \frac{d\langle N, M\rangle_{u}}{N_{u}}+\int_{\Lambda}^{t} \frac{d\langle N, M\rangle_{u}}{N_{u}-\bar{N}_{u}} .
\end{aligned}
$$

Now, the predictable representation property of $M$ yields $c \in \mathbb{R}$ and an $\mathbb{F}$-predictable process $n=\left(n_{t}\right)_{t \geq 0}$ such that

$$
N_{t}=c+\int_{0}^{t} n_{u} d M_{u}
$$

that is,

$$
d\langle N, M\rangle_{t}=n_{t} d\langle M, M\rangle_{t} .
$$

Substituting this into (3.1) we get

$$
\begin{aligned}
M_{t} & =\widehat{M}_{t}+\int_{0}^{t \wedge \Lambda} \frac{n_{u} d\langle M, M\rangle_{u}}{N_{u}}+\int_{\Lambda}^{t} \frac{n_{u} d\langle M, M\rangle_{u}}{N_{u}-\bar{N}_{u}} \\
& =\widehat{M}_{t}+\int_{0}^{t}\left(1_{[0, \Lambda]} \frac{n_{u}}{N_{u}}+1_{(\Lambda, T]} \frac{n_{u}}{N_{u}-\bar{N}_{u}}\right) d\langle M, M\rangle_{u} \\
& =: \widehat{M}_{t}+\int_{0}^{t} \widehat{\alpha}_{u} d\langle M, M\rangle_{u} .
\end{aligned}
$$

Since $\langle M, M\rangle=\langle\widehat{M}, \widehat{M}\rangle$, the decomposition of $S$ under the filtration $\mathbb{G}$ takes the form

$$
S_{t}=\widehat{M}_{t}+\int_{0}^{t}\left(\widehat{\alpha}_{u}+\alpha_{u}\right) d\langle\widehat{M}, \widehat{M}\rangle_{u} .
$$

Note that since $\alpha$ is $\mathbb{F}$-predictable, it is also $\mathbb{G}$-predictable, so that $\widetilde{\alpha}:=$ $\widehat{\alpha}_{u}+\alpha_{u}$ is $\mathbb{G}$-predictable. Therefore, in view of Theorem 2.2, if we can prove 
that $\mathbb{P}\left(\int_{0}^{T}\left(\alpha_{u}+\widehat{\alpha}_{u}\right)^{2} d\langle M, M\rangle_{u}=\infty\right)>0$, then there is no local martingale measure for $S$. First, observe that elementary inequalities give

$$
\begin{aligned}
& \int_{0}^{T}\left(\alpha_{u}+\widehat{\alpha}_{u}\right)^{2} d\langle M, M\rangle_{u} \\
&=\int_{0}^{T}\left\{\alpha_{u}^{2}+\left(\frac{n_{u}}{N_{u}}\right)^{2} 1_{[0, \Lambda]}+\left(\frac{n_{u}}{N_{u}-\bar{N}_{u}}\right)^{2} 1_{(\Lambda, T]}\right. \\
&\left.+2 \alpha_{u} \frac{n_{u}}{N_{u}} 1_{[0, \Lambda]}+2 \alpha_{u} \frac{n_{u}}{N_{u}-\bar{N}_{u}} 1_{(\Lambda, T]}\right\} d\langle\widehat{M}, \widehat{M}\rangle_{u} \\
&= \int_{0}^{T}\left\{\left(\alpha_{u}+1_{[0, \Lambda]} \frac{n_{u}}{N_{u}}\right)^{2}+\left(\frac{1}{2} \frac{n_{u}}{N_{u}-\bar{N}_{u}} 1_{(\Lambda, T]}+2 \alpha_{u}\right)^{2}\right. \\
&\left.-4 \alpha_{u}^{2}+\frac{3}{4}\left(\frac{n_{u}}{N_{u}-\bar{N}_{u}}\right)^{2} 1_{(\Lambda, T]}\right\} d\langle\widehat{M}, \widehat{M}\rangle_{u} \\
& \geq \int_{0}^{T}\left\{\frac{3}{4}\left(\frac{n_{u}}{N_{u}-\bar{N}_{u}}\right)^{2} 1_{(\Lambda, T]}-4 \alpha_{u}^{2}\right\} d\langle\widehat{M}, \widehat{M}\rangle_{u} \\
&= \int_{0}^{T}\left\{\frac{3}{4}\left(\frac{n_{u}}{N_{u}-\bar{N}_{u}}\right)^{2} 1_{(\Lambda, T]}-4 \alpha_{u}^{2}\right\} d\langle M, M\rangle_{u} .
\end{aligned}
$$

Since $S$ satisfies (NFLVR) under the filtration $\mathbb{F}$, we know that $\int_{0}^{T} \alpha_{u}^{2} d\langle M, M\rangle_{u}$ is $\mathbb{P}$-a.s. finite, and thus it suffices to show that $I_{\Lambda}:=\int_{\Lambda}^{T}\left(\frac{n_{u}}{N_{u}-\bar{N}_{u}}\right)^{2} d\langle M, M\rangle_{u}$ is infinite on a set with positive probability. Since by Theorem 2.6, $\Lambda=$ $\sup \left\{t: N_{t}=\bar{N}_{t}\right\}=\sup \left\{t: N_{t}=\bar{N}_{\infty}\right\}$, we have, for $u \geq \Lambda$,

$$
N_{u}-\bar{N}_{u}=N_{u}-\bar{N}_{\Lambda}=N_{u}-N_{\Lambda}=\int_{\Lambda}^{u} n_{s} d M_{s}
$$

and consequently

$$
I_{\Lambda}=\int_{\Lambda}^{T}\left(\frac{n_{u}}{\int_{\Lambda}^{u} n_{s} d M_{s}}\right)^{2} d\langle M, M\rangle_{u}
$$

Define now

$$
A=\left\{\omega: \int_{\Lambda}^{T}\left(\frac{n_{u}}{N_{u}-N_{\Lambda}}\right)^{2} d\langle M, M\rangle_{u}<\infty\right\} .
$$

We may assume that $P(A)=1$ (otherwise the proof is finished). In particular, the integrals $\int_{\Lambda}^{T} \frac{n_{u}}{N_{u}-N_{\Lambda}} d M_{u}$ and $\int_{\Lambda}^{T} \frac{n_{u}}{N_{u}-N_{\Lambda}} d \widehat{M}_{u}$ are well defined. Now, let $X_{t}=N_{t}-N_{\Lambda}$, and let $\varepsilon>0$ be such that $T>\Lambda+\varepsilon \mathbb{P}$-a.s. From Itô's 
formula we obtain

$$
\begin{aligned}
\ln \left(N_{T}-N_{\Lambda}\right) & -\ln \left(N_{\Lambda+\varepsilon}-N_{\Lambda}\right)=\ln \left(X_{T}\right)-\ln \left(X_{\Lambda+\varepsilon}\right) \\
& =\int_{\Lambda+\varepsilon}^{T} \frac{d X_{u}}{X_{u}}-\frac{1}{2} \int_{\Lambda+\varepsilon}^{T} \frac{d\langle X, X\rangle_{u}}{X_{u}^{2}} \\
& =\int_{\Lambda+\varepsilon}^{T} \frac{d N_{u}}{N_{u}-N_{\Lambda}}-\frac{1}{2} \int_{\Lambda+\varepsilon}^{T} \frac{d\langle N, N\rangle_{u}}{\left(N_{u}-N_{\Lambda}\right)^{2}} \\
& =\int_{\Lambda+\varepsilon}^{T} \frac{n_{u}}{N_{u}-N_{\Lambda}} d M_{u}-\frac{1}{2} \int_{\Lambda+\varepsilon}^{T}\left(\frac{n_{u}}{N_{u}-N_{\Lambda}}\right)^{2} d\langle M, M\rangle_{u} .
\end{aligned}
$$

Letting $\varepsilon \rightarrow 0$ we see that the integrals

$$
\int_{\Lambda}^{T} \frac{n_{u}}{N_{u}-N_{\Lambda}} d M_{u} \text { and } \int_{\Lambda}^{T}\left(\frac{n_{u}}{N_{u}-N_{\Lambda}}\right)^{2} d\langle M, M\rangle_{u}
$$

cannot be simultaneously finite on any set of positive probability. Since for $u \geq \Lambda$,

$$
M_{u}=\widehat{M}_{u}+\int_{0}^{\Lambda} \frac{n_{s}}{N_{s}} d\langle M, M\rangle_{s}+\int_{\Lambda}^{u} \frac{n_{s}}{N_{s}-N_{\Lambda}} d\langle M, M\rangle_{s},
$$

we may then write

$$
\int_{\Lambda}^{T} \frac{n_{u}}{N_{u}-N_{\Lambda}} d M_{u}=\int_{\Lambda}^{T} \frac{n_{u}}{N_{u}-N_{\Lambda}} d \widehat{M}_{u}+\int_{\Lambda}^{T}\left(\frac{n_{u}}{N_{u}-N_{\Lambda}}\right)^{2} d\langle M, M\rangle_{u},
$$

which shows that

$$
A \cap\left\{\int_{\Lambda}^{T} \frac{n_{u}}{N_{u}-N_{\Lambda}} d M_{u}=\infty\right\}=A \cap\left\{\int_{\Lambda}^{T} \frac{n_{u}}{N_{u}-N_{\Lambda}} d \widehat{M}_{u}=\infty\right\} .
$$

But $\widehat{M}$ is a $(\mathbb{G}, \mathbb{P})$-continuous local martingale, so that the time change formula for Brownian motion yields

$$
\int_{\Lambda}^{T} \frac{n_{u}}{N_{u}-N_{\Lambda}} d \widehat{M}_{u}=B_{U_{T}}
$$

where

$$
U_{T}=\int_{\Lambda}^{T}\left(\frac{n_{u}}{N_{u}-N_{\Lambda}}\right)^{2} d\langle\widehat{M}, \widehat{M}\rangle_{u}=\int_{\Lambda}^{T}\left(\frac{n_{u}}{N_{u}-N_{\Lambda}}\right)^{2} d\langle M, M\rangle_{u},
$$

and $B$ is some $(\mathbb{H}, \mathbb{P})$-Brownian motion, where $\mathbb{H}$ is a filtration resulting from the change of time. This shows that on the set $A$ we have $\int_{\Lambda}^{T} \frac{n_{u}}{N_{u}-N_{\Lambda}} d \widehat{M}_{u}$ 
$<\infty$, so that (3.2) reduces to

$$
A \cap\left\{\int_{\Lambda}^{T} \frac{n_{u}}{N_{u}-N_{\Lambda}} d M_{u}=\infty\right\}=\emptyset,
$$

or equivalently,

$$
A \subset\left\{\int_{\Lambda}^{T} \frac{n_{u}}{N_{u}-N_{\Lambda}} d M_{u}<\infty\right\} .
$$

Therefore $A$ must be an empty set, which contradicts the assumption $P(A)$ $=1$. Consequently, $\int_{0}^{T} 1_{(\Lambda, T]}\left(\frac{n_{u}}{N_{u}-N_{\Lambda}}\right)^{2} d\langle M, M\rangle_{u}$ is infinite on the set of positive probability. This ends the proof.

In fact, the above proof shows that the insider has an immediate arbitrage opportunity after time $\Lambda$. Let us briefly recall the definition of this notion. For details see [4].

Definition 3.2. An $(\mathbb{F}, \mathbb{P})$-semimartingale $S=\left(S_{t}\right)_{t \geq 0}$ admits immediate arbitrage at the $\mathbb{F}$-stopping time $T$ (we assume that $\mathbb{P}(T<\infty)>0$ ) if there exists an $S$-integrable strategy $H=\left(H_{t}\right)_{t \geq 0}$ such that $H_{t}=H_{t} 1_{] T, \infty]}(t)$ and $\int_{0}^{t} H_{u} d S_{u}>0$ for $t>T$.

Now, the proof of Theorem 3.1 shows that the Doob-Meyer decomposition of $S$ with respect to the enlarged filtration, $S_{t}=\widehat{M}_{t}+\int_{0}^{t} \widetilde{\alpha}_{u} d\langle\widehat{M}, \widehat{M}\rangle_{u}$, has the property that for each $\varepsilon>0, \int_{\Lambda}^{\Lambda+\varepsilon} \widetilde{\alpha}_{u}^{2} d\langle\widehat{M}, \widehat{M}\rangle_{u}$ is not finite $\mathbb{P}$-a.s. Thus, by applying Theorems 3.6 and 3.7 from [4] we immediately see that the insider has an immediate arbitrage opportunity after $\mathbb{G}$-stopping time $\Lambda$.

REMARK 3.3. The multiplicative decomposition of the Azéma supermartingale $Z_{t}=N_{t} / \bar{N}_{t}$ provides an interesting duality between initial and progressive enlargement. Namely, it is easy to calculate that initial enlargement by the value of $\bar{N}_{\infty}$, and progressive enlargement by honest time $\Lambda=\sup \left\{t: N_{t}=\bar{N}_{t}\right\}$ yield the same Doob-Meyer decomposition. Thus, it would be tempting to treat the insider progressive enlargement problems by methods of initial enlargement, which have been much better developed, in the insider trading context. Unfortunately, it is hard to construct $N=\left(N_{t}\right)_{t \geq 0}$ satisfying the conditions of Theorem 2.5 such that $\bar{N}_{\infty}$ is one of the random variables usually considered in the initial enlargement for the insider.

Nonetheless, we can use the above observation to obtain easily, within the framework of Theorem 3.1, some weaker result relating to the additional logarithmic utility of the insider. Using the fact that $\bar{N}_{\infty}$ has the same distribution as $1 / U$, where $U$ is a random variable with uniform distribution on $[0,1]$ (see Lemma 0.1 in [8]), and switching to the initial enlargement by 
$\sigma\left(\bar{N}_{\infty}\right)$, it follows immediately from Theorem 5.14 in [1] that the logarithmic utility increment, or equivalently $\mathbb{E} \int_{0}^{T} \widetilde{\alpha}_{u}^{2} d\langle\widehat{M}, \widehat{M}\rangle_{u}$, is infinite. However, $\mathbb{E} \int_{0}^{T} \widetilde{\alpha}_{u}^{2} d\langle\widehat{M}, \widehat{M}\rangle_{u}=\infty$ does not imply that $\mathbb{P}\left(\int_{0}^{T} \widetilde{\alpha}_{u}^{2} d\langle\widehat{M}, \widehat{M}\rangle_{u}=\infty\right)>0$, so that Theorem 3.1 is slightly stronger.

4. Arbitrage opportunities. We give an example of an honest time which also allows one easily to construct an arbitrage opportunity (resulting from the lack of equivalent local martingale measure).

EXAMPLE 4.1. Let $\Lambda_{T_{a}}=\sup \left\{u \leq T_{a}: B_{u}=0\right\}$ where $T_{a}=\inf \{t$ : $\left.B_{t}=a\right\}, a>0$ and $B=\left(B_{t}\right)_{t \geq 0}$ is a standard $(\mathbb{F}, \mathbb{P})$-Brownian motion. Clearly, $\Lambda_{T_{a}}$ is an honest time. Now, if the stock price process is e.g. a geometric Brownian motion with $\mu=0, \sigma=1$, that is, $S_{t}=\exp \left(B_{t}-\frac{1}{2} t\right)$, then buying $S$ at $\Lambda_{T_{a}}$ and selling at $T_{a} \wedge T$ will give sure profit, and thus it is an arbitrage opportunity. In this case the nonexistence of an equivalent local martingale measure in the enlarged filtration can also be easily deduced from the usual decomposition of $B$ :

$$
B_{t}=\widehat{B}_{t}-\int_{0}^{t \wedge \Lambda_{T_{a}}} \frac{1_{\left\{B_{s}>0\right\}}}{a-B_{s}} d s+\int_{\Lambda_{T_{a}}}^{t \wedge T_{a}} \frac{d s}{B_{s}}
$$

and the fact that $B_{\Lambda_{T_{a}}}=0$ and therefore for all $\varepsilon>0, \int_{\Lambda_{T_{a}}}^{\Lambda_{T_{a}}+\varepsilon} d s / B_{s}^{2}=\infty$ P-a.s.

Acknowledgements. I am grateful to Marc Yor for carefully reading the first version of this paper and many useful comments, and to the anonymous referee for corrections and comments which improved the paper.

\section{References}

[1] S. Ankirchner, S. Dereich and P. Imkeller, The Shannon information of filtrations and the additional logarithmic utility of insiders, Ann. Probab. 34 (2006), 743-778.

[2] S. Ankirchner and P. Imkeller, Finite utility on financial markets with asymmetric information and structure properties of the price dynamics, Ann. Inst. H. Poincaré Probab. Statist. 41 (2005), 479-503.

[3] F. Delbaen and W. Schachermayer, A general version of the fundamental theorem of asset pricing, Math. Ann. 300 (1994), 463-520.

[4] - - - The existence of absolutely continuous local martingale measures, Ann. Appl. Probab. 5 (1995), 926-945.

[5] P. Imkeller, Random times at which insiders can have free lunches, Stoch. Stoch. Rep. 74 (2002), 465-487.

[6] T. Jeulin, Semi-martingales et grossissement de filtration, Lecture Notes in Math. 833, Springer, 1980.

[7] T. Jeulin et M. Yor, Grossissements de filtrations : exemples et applications, Lecture Notes in Math. 1118, Springer, 1985. 
[8] R. Mansuy and M. Yor, Random Times and Enlargements of Filtrations in a Brownian Setting, Lecture Notes in Math. 1873, Springer, 2006.

[9] A. Nikeghbali and M. Yor, Doob's maximal identity, multiplicative decomposition and enlargements of filtrations, Illinois J. Math. 50 (2005), 791-814.

Jakub Zwierz

Mathematical Institute

Wrocław University

Pl. Grunwaldzki 2/4

50-384 Wrocław, Poland

E-mail: zwierz@math.uni.wroc.pl

Received January 2, 2007;

received in final form May 9, 2007 